\title{
An autosomal locus controls sex reversal in interspecific $X Y$ hybrids of the medaka fishes
}

\author{
M Kato ${ }^{1}$, Y Takehana ${ }^{2}$, Y Fukuda ${ }^{1}$, K Naruse $^{2}$, M Sakaizumi $^{1}$ and S Hamaguchi ${ }^{1}$ \\ ${ }^{1}$ Graduate School of Science and Technology, Niigata University, Niigata, Japan and ${ }^{2}$ Laboratory of Bioresources, National Institute for \\ Basic Biology, Okazaki, Japan
}

\begin{abstract}
Although the two medaka species Oryzias latipes and $O$. curvinotus share the sex-determining gene Dmy, $\mathrm{XY}$ sex reversal occurs in interspecific hybridization between $O$. latipes females of the Hd-rR inbred strain and $O$. curvinotus males. In this Hd-rR-curvinotus mating, all $X X$ and $X Y$ hybrids developed as females. In this study, we used another $O$. latipes inbred strain $(\mathrm{HNI})$ for the mating, and found that $23 \%$ of $X Y$ hybrids developed as males, although all $X X$ and the remaining $X Y$ hybrids developed as females. Linkage analysis using $236 \mathrm{XY}$ hybrid males obtained from ( $\mathrm{Hd}-$ $\mathrm{rR} \times \mathrm{HNI}) \mathrm{F}_{1}$ females showed that a single major locus, Hybrid maleless $(\mathrm{Hm})$, on autosomal linkage group 17, contributed to the strain difference in the $X Y$ sex reversal. Furthermore, we found that crossing females of a different $O$.
\end{abstract}

latipes inbred strain, $\mathrm{HO} 4 \mathrm{C}$, did not cause $\mathrm{XY}$ sex reversal in the interspecific hybrids, and that the $X Y$ hybrids from $(\mathrm{Hd}$ $\mathrm{rR} \times \mathrm{HO} 4 \mathrm{C}) \mathrm{F}_{1}$ females showed a 1:1 sex ratio. $X Y$ hybrid males had the $\mathrm{HO} 4 \mathrm{C}$ allele at sequence-tagged site loci around the $\mathrm{Hml}$ locus whereas $\mathrm{XY}$ females had the Hd-rR allele, confirming the strong contribution of this locus to $X Y$ sex reversal. Reverse transcriptase PCR analysis showed a reduced expression of Dmycurvinotus in XY fry of the Hd-rRcurvinotus hybrids at hatching. These results suggest that the Hd-rR allele at the $\mathrm{Hml}$ locus interfere with the function of Dmycurvinotus on a hybrid background, thus resulting in $\mathrm{XY}$ sex reversal.

Heredity (2011) 107, 523-529; doi:10.1038/hdy.2011.38; published online 18 May 2011

Keywords: Dmy, hybrids; medaka; sex-determining gene; sex reversal

\section{Introduction}

Gonadal sex determination is a fundamental process in vertebrates because the phenotypic sex depends on whether the gonad develops into a testis or an ovary. In most mammals, a single sex-determining gene on the $Y$ chromosome, SRY is sufficient to induce the undifferentiated gonad to develop as a testis (Gubbay et al., 1990; Sinclair et al., 1990; Koopman et al., 1991; Capel et al., 1993). SRY encodes a high mobility group domain transcription factor, and its downstream target is the Sox9 gene (Sekido and Lovell-Badge, 2008). Many other key downstream genes have been identified as having roles in the sex-differentiation pathway in human and mouse, although their molecular functions and the regulatory networks among these genes are unknown (Wilhelm et al., 2007).

A second vertebrate sex-determining gene was identified in the medaka fish, Oryzias latipes. This species also has an XX/XY sex-determination system (Aida, 1921), but initiation of the male-determination pathway begins with the expression of another $\mathrm{Y}$ chromosome-specific gene, Dmy (Matsuda et al., 2002). Dmy is the Y-specific paralog of the autosomal Dmrt1 gene which appears to

Correspondence: Dr S Hamaguchi, Graduate School of Science and Technology, Niigata University, 8050 Ikarashi-2, Nishi-ku, Niigata 9502181, Japan.

E-mail: shamaguc@env.sc.niigata-u.ac.jp

Received 5 October 2010; revised 2 February 2011; accepted 21 March 2011; published online 18 May 2011 be involved in male sexual development in vertebrates (Nanda et al., 2002). Mutations with impaired Dmy function cause XY sex reversal (Shinomiya et al., 2004; Otake et al., 2006, 2008) and the presence of an exogenous Dmy gene induces XX sex reversal (Matsuda et al., 2007; Otake et al., 2009), suggesting that this gene is necessary and sufficient to induce testicular development. Dmy contains a DNA-binding motif (the DM domain), and is thus thought to encode a putative transcription factor that activates or represses downstream target genes to regulate sexual differentiation of the bipotential gonad. However, it remains unclear how Dmy controls the maledetermination pathway and whether its downstream genes are conserved among vertebrates.

A straightforward approach for identifying genes involved in the sex-determination pathway is the genetic analyses of sex-reversal conditions. Our previous study demonstrated an intriguing sex reversal in the interspecific hybridization between $O$. latipes and $O$. curvinotus (Shinomiya et al., 2006), despite these two species having a common sex-determining pathway with the Dmy gene on the homologous Y chromosome (Matsuda et al., 2003). In XYlatipes hybrids between O. curvinotus females and $O$. latipes males, $\mathrm{Hd}-\mathrm{rR}$ strain males produced XY females in the hybrids (110 males and 30 females), whereas HNI males produced no XY females (70 males and 0 females). This demonstrates that there is a strain difference in the incidence of XYlatipes females (Shinomiya et al., 2006; Kato et al., 2010). A congenic approach successfully demonstrated that a small region on the $\mathrm{Y}$ chromosome, which included Dmy, was 
responsible for the strain-specific difference in this XYlatipes sex reversal (Kato et al., 2010).

In the opposite cross between O. latipes females (Hd-rR strain) and $O$. curvinotus males, all $X Y^{\text {curvinotus }}$ hybrids developed as females. This suggests that Dmy derived from O. curvinotus cannot determine maleness in the hybrid background (Shinomiya et al., 2006). A previous study reported that $O$. latipes females from some wild strains produced both males and females in the interspecific hybrids (Sakaizumi et al., 1992), implying that there were genetic background differences in the incidence of the XYcurvinotus sex reversal. However, these strain differences have not been confirmed because there were no sex chromosome-linked markers available in the hybrid fish until recently.

In the present study, we compared the ability to induce maleness in the XYcurvinotus hybrids among three medaka inbred strains, Hd-rR, HNI and HO4C, using the Dmy gene as a $\mathrm{Y}$ chromosomal marker. Because these strains showed different incidences of $X Y$ sex reversal in the interspecific hybrids, we mapped the chromosomal region responsible for the sex reversal. Linkage analysis using the Hd-rR and HNI strains identified a single major locus on linkage group (LG) 17, which was associated with the strain differences in the $X Y$ sex reversal. This region also contributed to differences in the frequency of hybrid sex reversal between the Hd-rR and HO4C inbred strains, suggesting that the hybrid XY sex reversal may be caused by an incompatibility between a single autosomal locus derived from $O$. latipes and the Dmy allele of O. curvinotus. Further analysis will reveal the molecules that interact with Dmy, and allow us to understand the molecular mechanisms of sex determination that begin with Dmy.

\section{Materials and methods}

\section{Fish}

We used a laboratory stock of $O$. curvinotus, as well as three inbred strains (HNI, Hd-rR and HO4C) of O. latipes. All fish were supplied by a sub-center (Niigata University, Japan) of the National Bioresource Project (Medaka) (http://www.shigen.nig.ac.jp/medaka/). The wild stock of O. curvinotus was originally collected in Hong Kong in 1986 (see Takehana et al., 2005). HNI was established from the Northern Japanese Population, whereas Hd-rR and $\mathrm{HO} 4 \mathrm{C}$ were from the Southern Japanese Population (Hyodo-Taguchi, 1996; Kinoshita et al., 2009). The fish were maintained in aquaria under an artificial 14-h light/10-h dark photoperiod at $27 \pm 2{ }^{\circ} \mathrm{C}$.

\section{Mating and sexing}

Hybrid fish were obtained from matings between $O$. latipes females and $O$. curvinotus males (XY or $\mathrm{YY}$ males) by pair mating. Naturally spawned eggs were collected and incubated under the same conditions as the adult fish. Hatched hybrid fish were reared until maturation for 2-3 months, and examined for their phenotypic and genotypic sexes.

Phenotypic sex was judged based on secondary sex characteristics, namely, the shapes of the dorsal and anal fins. Genotypic sex $(X Y$ or $X X)$ was determined by the presence or absence of the Dmy gene of $O$. curvinotus evaluated by PCR amplification of caudal fin clip genomic DNA extracted from adult fish. PCR amplification was performed with the primers PG17.eS (5'-CGCCTTGAGGAGGCAGCAGG-3') and PG17.20U (5'-GCATCTGCTGGTACTGCTGGTAGTTG-3') with an annealing temperature of $65^{\circ} \mathrm{C}$ (Shinomiya et al., 2006). Because the PCR primers amplify both Dmy and its autosomal paralog Dmrt1, individuals with only Dmrt1 fragments were judged to have an XX genotype, and those with both Dmy and Dmrt1 fragments were judged to have an $\mathrm{XY}$ genotype.

\section{Production of $\mathrm{YY}$ males}

Fertilized eggs of $O$. curvinotus were incubated in water containing estradiol-17 $\beta$ (Sigma, St Louis, MO, USA) at $0.2 \mu \mathrm{g} \mathrm{ml}^{-1}$ until hatching. Hatched fry were transferred to normal tap water and fed on a commercial pet-food diet until sexual maturation. Sex-reversed $X Y$ females were identified by PCR genotyping of Dmy and subsequently mated with normal $X Y$ males. $Y Y$ males were selected by PCR genotyping of Dmy and Casp6 (Kondo et al., 2001; Matsuda et al., 2003).

\section{Genetic mapping}

Sixty expressed sequence tag (EST) markers covering twenty-four chromosomes were used to map the locus responsible for strain-specific differences in hybrid sex reversal. The primer sequences used in this study are listed in Supplementary Table S1, and the mapped positions of the EST markers are available in M Base (http:/ / earth.lab.nig.ac.jp/ mbase/medaka_top.html). In addition, we designed sequence-tagged site (STS) primers based on genome sequence data (UTGB medaka genome browser: http://medaka.utgenome.org/ or Ensembl genome browser: http://www.ensembl.org), and used these STS markers for fine mapping. Primer sequences and chromosomal locations of these markers are listed in Supplementary Table S2. Individual PCR conditions were optimized for each primer pair, and products were digested with restriction enzymes if necessary. Separation of PCR products was performed by conventional polyacrylamide gel electrophoresis (Kimura et al., 2004).

\section{RNA extraction and RT-PCR}

Total RNA was extracted from fry at 8 days post fertilization (dpf) using an RNeasy Mini Kit (Qiagen, Tokyo, Japan), and subjected to reverse transcriptase PCR (RT-PCR) using a OneStep RT-PCR Kit (Qiagen). Aliquots (20 ng) of the total RNA were used as templates in $25 \mu \mathrm{l}$ reaction volumes. The PCR amplification conditions were as follows: $30 \mathrm{~min}$ at $55^{\circ} \mathrm{C}$; $15 \mathrm{~min}$ at $95^{\circ} \mathrm{C}$; cycles of $30 \mathrm{~s}$ at $96^{\circ} \mathrm{C}, 30 \mathrm{~s}$ at the annealing temperature and $60 \mathrm{~s}$ at $72^{\circ} \mathrm{C}$; and $5 \mathrm{~min}$ at $72{ }^{\circ} \mathrm{C}$. The number of PCR cycles for Dmy and $\beta$-actin was adjusted to be 36 cycles and 22 cycles, and the annealing temperature at $63^{\circ} \mathrm{C}$ and $55^{\circ} \mathrm{C}$, respectively. The primers for Dmy (cY331, 5'-AGG CTT CGT CCG GCC CTG AA-3' and cY4-5U, 5'-GAG GCT CCT GGT GCA GAA CG-3') amplified a 449-bp DNA fragment. The primers for $\beta$ actin (3b, 5'-CMG TCA GGA TCT TCA TSA GG-3' and 4, 5'-CAC ACC TTC TAC AAT GAG CTG A-3') amplified a 322-bp DNA fragment (Otake et al., 2006). Aliquots of 8 and $2 \mu \mathrm{l}$ of the Dmy and $\beta$-actin RT-PCR products, respectively, were electrophoresed in a $2 \%$ agarose gel and stained with ethidium bromide. 


\section{Results}

The $\mathrm{HNI}$ strain produces $\mathrm{XY}$ males in the hybrids Our previous study indicated that interspecific hybridization between $O$. latipes females and $O$. curvinotus males caused complete XY sex reversal (Shinomiya et al., 2006). We used Hd-rR inbred strain females for matings with $O$. curvinotus males (Hd-rR-curvinotus), and found that all 569 hybrids developed as females. Among 99 genotyped individuals, 55 individuals had the XX genotype and 44 had $X Y$, demonstrating that all $X Y$ individuals were sex reversed (Table 1). In the present study, we used another inbred strain, $\mathrm{HNI}$, for matings with O. curvinotus males (HNI-curvinotus). All 69 XX hybrids were female, whereas the $60 \mathrm{XY}$ hybrids consisted of 46 females and 14 males (23\%), indicating that the HNI strain could produce XY males in the hybrids. This means that the two inbred strains HNI and Hd-rR differ in their incidences of $X Y$ sex reversal in the interspecific hybrids. Next, we used (Hd$\mathrm{rR} \times \mathrm{HNI}) \mathrm{F}_{1}$ females and $\left(\mathrm{F}_{1} \times \mathrm{Hd}-\mathrm{rR}\right) \quad \mathrm{BC}_{1}$ females for matings with $O$. curvinotus males. The $\mathrm{F}_{1}$ females produced 147 females and 38 males (21\%) in the XY hybrids, and four out of five $\mathrm{BC}_{1}$ females produced males in the $\mathrm{XY}$ hybrids wheras the one remaining female did not (Table 1).

\section{A single major locus controls the sex reversal ratio in the $X Y$ hybrids}

To map the chromosomal region(s) responsible for the strain differences in the $\mathrm{XY}$ sex reversal ratio, an initial linkage analysis was performed using EST markers established in $O$. latipes. In the cross between $(\mathrm{Hd}-\mathrm{rR} \times \mathrm{HNI}) \mathrm{F}_{1}$ females and $\mathrm{O}$. curvinotus males, the hybrids have either an Hd-rR- or an HNI-derived allele at each locus (Figure 1). Because XY males were found only in the HNI-curvinotus hybrids, we assumed that the chromosomal regions heterozygous for HNI and $O$. curvinotus were responsible for producing $X Y$ males. Among 38 hybrid males obtained from the cross, we selected 24 males and used them for genome-wide genotyping with 60 EST markers covering 24 chromosomes. Then we searched for loci that showed a significant deviation from the expected 1:1 ratio, using a classical chi-squared test. The tests revealed significantly high chi-squared values at four loci, OLa21.11f, OLc30.09h, AU168385 and MF01SSA074C05 $(P<0.01)$, which are all located on LG 17 (Table 2). At these loci, $\mathrm{HNI} /$ curvinotus heterozygotes appeared at a significantly higher frequency than the 1:1 ratio, but other loci did not

Table 1 Genotypic and phenotypic sexes of hybrids between Oryzias latipes females and O. curvinotus males

\begin{tabular}{|c|c|c|c|c|}
\hline \multirow[t]{2}{*}{ Parental females } & \multirow{2}{*}{$\frac{\mathrm{XX}}{\text { Females }}$} & \multicolumn{2}{|c|}{$X Y$} & \multirow[t]{2}{*}{ Total } \\
\hline & & Females & Males (\%) & \\
\hline Hd-rR ${ }^{a}$ & 55 & 44 & $0(0)$ & 99 \\
\hline HNI & 69 & 46 & $14(23)$ & 129 \\
\hline$(\mathrm{Hd}-\mathrm{rR} \times \mathrm{HNI}) \mathrm{F}_{1}$ & 194 & 147 & $38(21)$ & 379 \\
\hline$\left(\mathrm{F}_{1} \times\right.$ Hd-rR $) \mathrm{BC}_{1} \# 1$ & 58 & 37 & $7(16)$ & 102 \\
\hline$\left(\mathrm{F}_{1} \times \mathrm{Hd}-\mathrm{rR}\right) \mathrm{BC}_{1} \# 2$ & 7 & 7 & 1 (13) & 15 \\
\hline$\left(\mathrm{F}_{1} \times \mathrm{Hd}-\mathrm{rR}\right) \mathrm{BC}_{1} \# 3$ & 26 & 14 & $2(13)$ & 42 \\
\hline$\left(\mathrm{F}_{1} \times\right.$ Hd-rR $) \mathrm{BC}_{1} \# 4$ & 46 & 42 & $9(18)$ & 97 \\
\hline$\left(\mathrm{F}_{1} \times\right.$ Hd-rR $) \mathrm{BC}_{1} \# 5$ & 30 & 29 & $0(0)$ & 59 \\
\hline
\end{tabular}

aSource: Shinomiya et al. (2006). show a significant deviation from the expected 1:1 segregation. Furthermore, genotyping for the $\mathrm{BC}_{1}$ females used in progeny tests (see Table 1 ) revealed that $\mathrm{BC}_{1}$ females producing $\mathrm{XY}$ males had the $\mathrm{HNI} / \mathrm{Hd}-\mathrm{rR}$ genotype whereas those producing only $\mathrm{XY}$ females had the Hd-rR/Hd-rR genotype at the EST markers on LG 17 (data not shown). These results suggested that a single major locus, which we named Hybrid maleless $(\mathrm{Hml})$, was associated with the strain differences in the hybrid sex reversal ratio.

Genetic mapping using all $38 \mathrm{XY}$ hybrid males showed that the $\mathrm{Hml}$ locus was located on LG 17 flanked by the two molecular markers AU171199 and LG17-29 (Figure 2a). To map the $\mathrm{Hml}$ locus precisely, we obtained an additional $198 \mathrm{XY}$ hybrid males from matings between (Hd-rR $\times \mathrm{HNI}$ ) $\mathrm{F}_{1}$ females and $\mathrm{O}$. curvinotus YY males, and genotyped new STS markers. This analysis narrowed the chromosomal location of $\mathrm{Hml}$ to a $1.2 \mathrm{cM}$ interval between the two markers gs20859 and LG17-29, with strong linkage to an additional four markers LG17-54, sca3316, LG17-49 and LG17-51 (0 recombination events in 236 meioses; Figure 2b). On the basis of the latest genome assembly (version 1.0), three scaffolds (354, 435 and 1423) were mapped to this region, although there remain large gaps (approximately

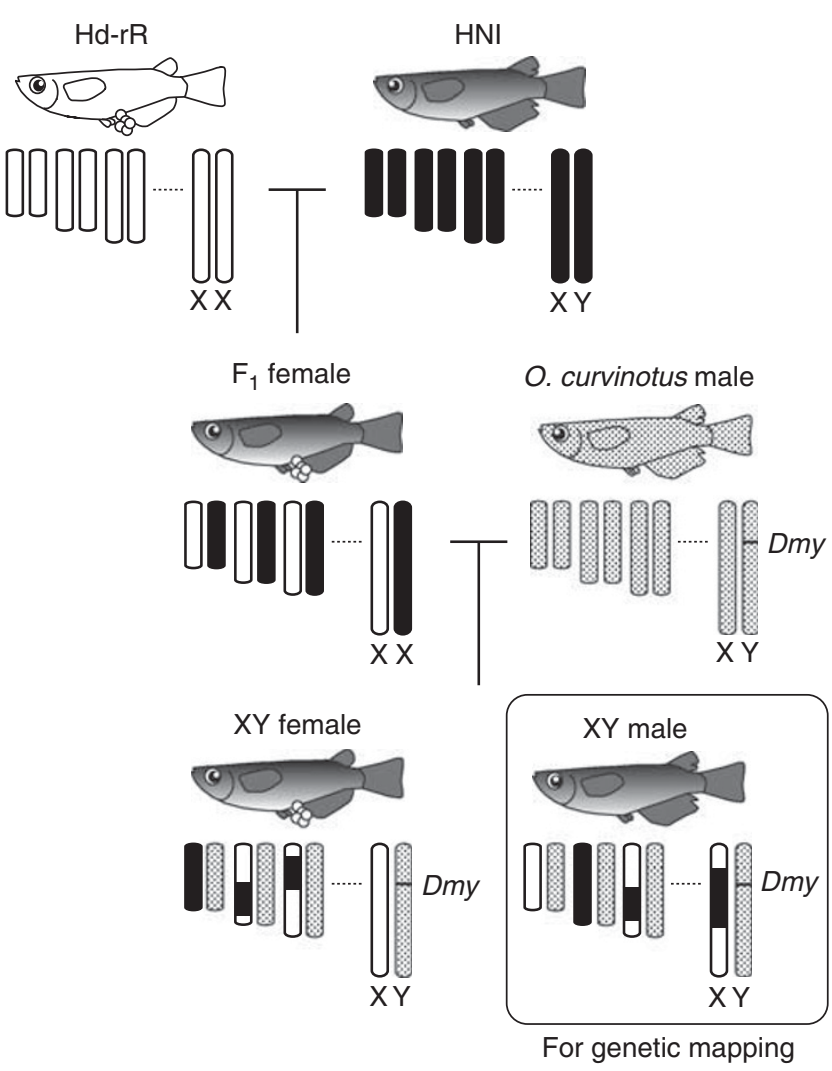

Figure 1 Mating scheme for linkage analysis. $F_{1}$ females between Hd-rR and HNI strains were crossed with O. curvinotus males, and the resulting $X Y$ males in the hybrids were used for genetic mapping. These $X Y$ males had an $O$. curvinotus-derived $Y$ chromosome and various combinations of HNI chromosomal segments in the autosomes and the $\mathrm{X}$ chromosome. We searched for chromosomal regions heterozygous for $\mathrm{HNI}$ and $O$. curvinotus alleles, which could produce XY males. Solid, HNI-derived chromosome; open, Hd-rR-derived chromosome; dotted, O. curvinotus-derived chromosome. The position of the sex-determining gene Dmy is shown. 
Table 2 EST markers associated with the strain difference in the XY sex reversal

\begin{tabular}{|c|c|c|c|c|c|c|c|}
\hline$L G$ & EST marker & Position (Mbp) & D/C genotype & N/C genotype & Total & $\chi^{2}$ value & $\mathrm{P}$ \\
\hline 17 & OLa21.11f & 3.4 & 4 & 18 & 22 & 8.91 & 0.0028 \\
\hline 17 & OLc30.09h & 4.9 & 3 & 20 & 23 & 12.6 & 0.00039 \\
\hline 17 & AU168385 & 13.4 & 1 & 22 & 23 & 19.2 & 0.000012 \\
\hline 17 & MF01SSA074C05 & 17.4 & 3 & 20 & 23 & 12.6 & 0.00039 \\
\hline
\end{tabular}

Abbreviation: EST, expressed sequence tag.

Of 60 markers throughout the genome, only significant markers are listed in the table (threshold value, $P<0.01, \mathrm{df}=1$ ). $\mathrm{D} / \mathrm{C}, \mathrm{heterozygous}$ for Hd-rR and O. curvinotus alleles; N/C, heterozygous for HNI and O. curvinotus alleles. Positions of the markers were retrieved from the medaka ensemble database (Ensembl release 57; http://www.ensembl.org/).

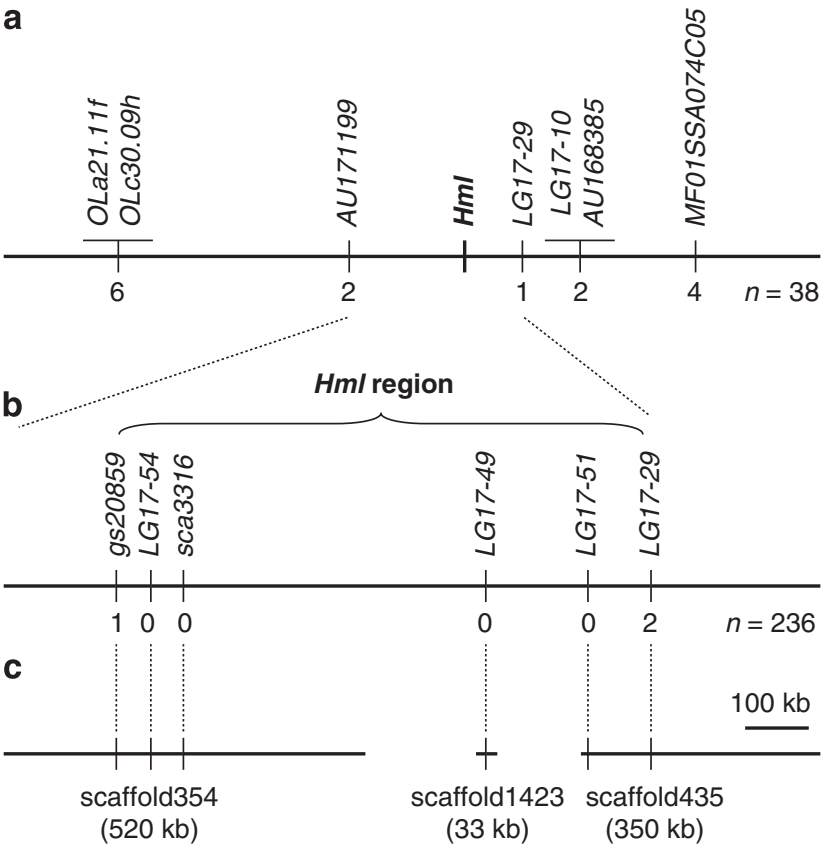

Figure 2 Genetic and physical map around the $H m l$ locus. (a) A sparse genetic map around the $\mathrm{Hml}$ locus on linkage group 17. (b) A high-resolution recombination map around the Hml locus. The $\mathrm{Hml}$ gene was mapped to a $1.2 \mathrm{~cm}$ interval based on the genotyping of $236 \mathrm{XY}$ hybrid males. The numbers under each map indicate the number of recombinants between $\mathrm{Hml}$ and the adjacent marker. (c) A physical map around the $\mathrm{Hml}$ locus based on the available genome sequence data (version 1.0; http://medaka. utgenome.org/). Three scaffolds (354, 435 and 1423) are mapped to this region but there remain gaps between the scaffolds.

120 and $140 \mathrm{~kb}$ ) between the scaffolds (Figure 2c). To find candidate genes in this region, we surveyed mapped genes on these scaffolds using the UCSC genome browser (http://genome.ucsc.edu). Among 12 genes found in this region (chr17: 10.8-11.6 Mb), seven encoded zinc finger proteins, suggesting a clustered organization of these genes on the $\mathrm{Hml}$ region.

\section{The HO4C strain did not cause $\mathrm{XY}$ sex reversal in the hybrids}

For further fine mapping, we used another inbred strain, $\mathrm{HO} 4 \mathrm{C}$, for matings with $\mathrm{YY}$ males of O. curvinotus (HO4C-curvinotus). In this cross, all $34 \mathrm{XY}$ hybrids developed as males with no sex reversal (Table 3), demonstrating that Dmy $y^{\text {curvinotus }}$ sufficiently induced maleness on this hybrid background. Then, we used
Table 3 Genotypic and phenotypic sexes of hybrids between Oryzias latipes females and O. curvinotus YY males

\begin{tabular}{lccr}
\hline Parental females & \multicolumn{2}{c}{$\mathrm{XY}$} & Total \\
\cline { 2 - 3 } & Females & Males (\%) & \\
\hline $\mathrm{HO} 4 \mathrm{C}$ & 0 & $34(100)$ & 34 \\
$(\mathrm{Hd}-\mathrm{rR} \times \mathrm{HO} 4 \mathrm{C}) \mathrm{F}_{1}$ & 121 & $135(53)$ & 256 \\
\hline
\end{tabular}

Table 4 Linkage analysis between phenotypic sex and STS markers on linkage group 17 in $\mathrm{XY}$ hybrids between $\left(\mathrm{Hd}-\mathrm{rR} \times \mathrm{HO}^{\mathrm{C}}\right) \mathrm{F}_{1}$ females and $O$. curvinotus males

\begin{tabular}{lcclccc}
\hline STS marker & \multicolumn{2}{c}{ D/C genotype } & & \multicolumn{2}{c}{ O/C genotype } & Total \\
\cline { 2 - 3 } & Females & Males & & Females & Males & \\
\hline gs20859 & 121 & 0 & & 0 & 135 & 256 \\
LG17-10 & 120 & 1 & & 1 & 134 & 256 \\
\hline
\end{tabular}

Abbreviation: STS, sequence-tagged site.

$\mathrm{D} / \mathrm{C}$, heterozygous for Hd-rR and O. curvinotus alleles; O/C, heterozygous for $\mathrm{HO} 4 \mathrm{c}$ and $\mathrm{O}$. curvinotus alleles.

$(\mathrm{Hd}-\mathrm{rR} \times \mathrm{HO} 4 \mathrm{C}) \mathrm{F}_{1}$ females for the matings, and found a 1:1 Mendelian ratio of males to females in the XY hybrids (135 and 121 progeny, respectively) (Table 3). Furthermore, two STS markers, gs20859 and LG17-10, showed strong linkage to the phenotypic sex (Table 4). For these loci, XY females had the Hd-rR/curvinotus genotype whereas $\mathrm{XY}$ males had the $\mathrm{HO} 4 \mathrm{C} /$ curvinotus genotype. These results strongly suggest that the $\mathrm{Hml}$ locus is involved in the different sex reversal ratios between HdrR-curvinotus and HO4C-curvinotus hybrids.

Reduced Dmy expression in Hd-rR-curvinotus XY hybrids Reduced expression levels of the sex-determining gene Dmy have been observed in some $\mathrm{XY}$ sex-reversal mutants of O. latipes (Matsuda et al., 2002; Otake et al., 2006). To examine the relationship between the hybrid sex reversal and the Dmy curvinotus expression level, we performed an RT-PCR analysis of fry at hatching ( $8 \mathrm{dpf})$ in the Hd-rR-curvinotus hybrids, the HO4C-curvinotus hybrids and the male parental strain of $O$. curvinotus (Figure 3). The Dmy mRNA level was slightly lower in $X Y$ fry of the HO4C-curvinotus hybrids than in that of $O$. curvinotus, although all the $X Y$ hybrids developed as males in adulthood. On the other hand, Dmy expression was not detected in $\mathrm{XY}$ fry of the Hd-rR-curvinotus hybrids, which developed as all females in adulthood, 

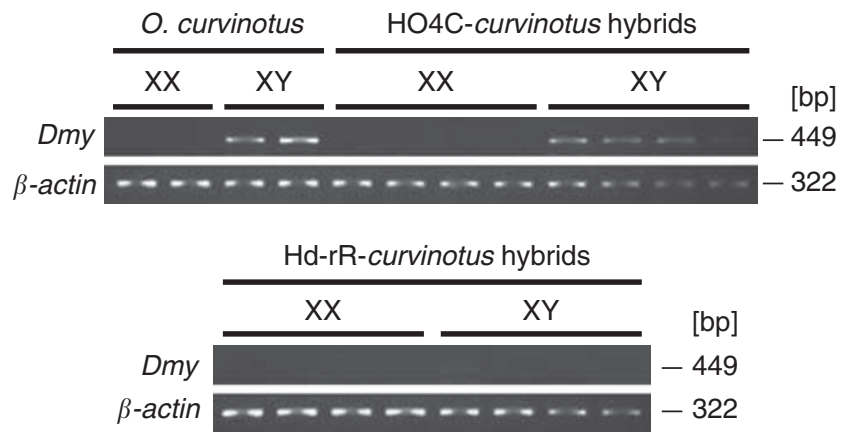

Figure 3 Expression of Dmy in the medaka hybrids. Dmy curvinotus mRNA expression in fry at hatching $(8 \mathrm{dpf})$ was analysed by RTPCR. $\beta$-actin expression was determined for calibration. Depressed or eliminated expression of Dmy transcripts was observed in XY fry of the Hd-rR-curvinotus hybrids.

suggesting that reduced expression of Dmy ${ }^{\text {curvinotus }}$ was associated with the $X Y$ sex reversal in the Hd-rRcurvinotus hybrids.

\section{Discussion}

Our mating experiments clearly demonstrated that the incidence of $\mathrm{XY}$ females was different among inbred strains of $O$. latipes. All XY individuals (44/44) in Hd-rRcurvinotus hybrids were sex-reversed females, whereas $23 \%(14 / 60)$ of XY HNI-curvinotus hybrids developed as males. Furthermore, all XY (34/34) HO4C-curvinotus hybrids developed as males, indicating that there are strain differences in the ability to induce maleness in the $\mathrm{XY}$ hybrids. Among these hybrids, the $\mathrm{Y}$ chromosome and one set of autosomes are derived from $O$. curvinotus whereas another set of autosomes and the $X$ chromosome are different, suggesting that the presence or absence of $X Y$ females in the hybrids can be attributed to the differences in the maternal genome. Notably, male fish appeared only in XY individuals, indicating that Dmy curvinotus is necessary for the male determination of the hybrids. Taken together, these findings suggest that the $X Y$ sex reversal in the hybrids results from incompatibility between the Dmy curvinotus $^{\text {allele }}$ and the $O$. latipes alleles of autosomal and/or $X$ chromosomal loci.

The sex reversal condition observed in the medaka hybrids is similar to $\mathrm{XY}$ sex reversal in B6- $\mathrm{Y}^{\mathrm{POS}}$ mice. This situation occurs when the $\mathrm{Y}$ chromosome derived from Mus poschiavinus ( $\mathrm{Y}^{\mathrm{POS}}$ ) is transferred onto the C57BL/6J (B6) inbred strain that normally carries a M. musculus $\mathrm{Y}$ chromosome (Eicher et al., 1982). Genetic background differences have been characterized in the inbred mouse strains. The B6 background was particularly sensitive to $\mathrm{XY}$ sex reversal, whereas other strains, including DBA/2J and 129S1/SvImJ, were found to be completely resistant to $\mathrm{Y}^{\mathrm{POS}}$-associated sex reversal (Nikolova et al., 2008). Quantitative trait loci mapping using the B6-YPOS and DBA/2J strains identified multiple loci that conferred some but not all of the observed sensitivity to $X Y$ sex reversal in B6 (Eicher et al., 1996). More recently, a congenic approach using the B6.129S1/SvImJ-Y ${ }^{\text {POS }}$ strain identified that a chromosome 11 region derived from the 129S1/SvImJ strain provided partial protection from sex reversal in XYPOS mice (Nikolova et al., 2008). These findings implicate the combined effects of many loci (rather than a single gene) in conferring the sensitivity to sex reversal in B6-Y $\mathrm{Y}^{\mathrm{POS}}$; this has been confirmed by a recent expression quantitative trait loci approach (Munger et al., 2009).

By contrast, a single locus is implicated in sex reversal in the medaka hybrids. (Hd-rR $\times \mathrm{HNI}$ ) $\mathrm{F}_{1}$ females produced $X Y$ males in the interspecific hybrids, and four out of five $\left(\mathrm{F}_{1} \times \mathrm{Hd}-\mathrm{rR}\right) \mathrm{BC}_{1}$ females produced $\mathrm{XY}$ males, whereas the remaining $\mathrm{BC}_{1}$ female did not, implying that a small number of genes contributed to the strain differences in the hybrid XY sex reversal. Our linkage analysis using $\mathrm{XY}$ hybrid males obtained from (Hd$\mathrm{rR} \times \mathrm{HNI}) \mathrm{F}_{1}$ females successfully identified the single major locus $\mathrm{Hml}$ on LG 17 that confers sensitivity to the $X Y$ sex reversal in the hybrids. In addition, genotyping for $X Y$ hybrids obtained from (Hd-rR $\times$ HO4C) $F_{1}$ females revealed strong linkage to STS markers around the Hml locus, suggesting that the same Hml locus contributed to the sex reversal in the different XY hybrids. These results suggest that a single gene, rather than a disrupted global network, causes XY sex reversal in the medaka hybrids. Thus, it is likely that allelic differences at the single $\mathrm{Hml}$ locus affect Dmy curvinotus function in the male-determining process in the hybrid fish.

Dmy transcripts first appear just before hatching exclusively in $X Y$ individuals of $O$. latipes and $O$. curvinotus (Matsuda et al., 2003; Kobayashi et al., 2004; Shinomiya et al., 2006). Reduced expression of the Dmy gene causes male-to-female sex reversal in O. latipes, suggesting that a threshold level of Dmy expression is required for male development (Matsuda et al., 2002; Otake et al., 2006, 2008). Our RT-PCR analysis of the medaka hybrids also revealed that Dmy expression levels in $\mathrm{XY}$ fry differed between Hd-rR-curvinotus and HO4C-curvinotus hybrids, in agreement with their sex reversal ratio. Hd-rR-curvinotus hybrids showed a loss of $D m y^{\text {curvinotus }}$ expression in the XY fry, which developed as all phenotypic females, suggesting that the $\mathrm{Hml}$ gene is an upstream regulator of Dmy. Thus, the XY sex reversal in the hybrids may result from an incompatibility between the cis-regulatory region of the Dmy $y^{\text {curvinotus }}$ allele and some $\mathrm{Hml}$ alleles of O. latipes as a trans-acting factor.

In this study, only $23 \%$ of $\mathrm{XY}$ individuals developed as males in the HNI-curvinotus hybrids. XY hybrids harboring identical genotypes developed as males or females, suggesting an incomplete penetrance for this phenotype. In the medaka mutants, a low level of Dmy expression below a threshold can induce sex reversal in a subset of $X Y$ individuals having the same chromosomal condition (Otake et al., 2006). Thus, a certain threshold level of Dmy expression may be required for male determination also in the hybrid backgrounds. Similar to the HNI-curvinotus hybrids, we obtained male $\mathrm{XY}$ hybrids from $(\mathrm{Hd}-\mathrm{rR} \times \mathrm{HNI}) \mathrm{F}_{1}$ females. However, the incidence of $X Y$ males $(21 \%)$ was higher than expected. In this cross, we estimated that approximately $11 \%$ of $X Y$ individuals would become males, because half of the XY hybrids would have the Hd-rR allele at the $\mathrm{Hml}$ locus, and develop as females, whereas the other half of the XY hybrids would have the HNI allele, and consist of males (23\%) and females (77\%). The observed high incidence of $\mathrm{XY}$ hybrid males is probably due to a 
background effect when the HNI allele at the Hml locus is in the hybrid background containing a part of the HdrR genome. Therefore, the Hd-rR background in the hybrids seems to affect the Dmy expression level itself or the threshold for Dmy expression, and thus cause the higher frequency of the XY males. However, further expression analysis of Dmy is necessary to test these hypotheses.

In both $O$. latipes and O. curvinotus, the first appearance of morphological sex differentiation was a difference in the number of germ cells between $X X$ and $X Y$ embryos, and a subset of germ cells in $\mathrm{XX}$ entered meiosis around hatching (Shinomiya et al., 2006). Later, morphological sex differences in somatic cells were observed. When Dmy function is impaired, germ cells in XY embryos start to proliferate and then enter meiosis just like XX embryos (Otake et al., 2006, 2008). These findings suggest that Dmy is involved in the regulation of germ cell proliferation at the early sex-determining stage and the formation of the testicular architecture. Previous histological observation of developing gonads in Hd-rR-curvinotus hybrids demonstrated that active proliferation of germ cells and oogenesis occurred in early gonadal development in the $X Y$ hybrids, identical to the early ovarian development in the XX hybrids (Shinomiya et al., 2006). Therefore, it is likely that the mismatching of the Hd-rR allele at the $\mathrm{Hml}$ locus with the regulatory region of Dmy curvinotus in the hybrids causes reduced expression of Dmy and disruption of the initial testis-determination steps including suppression of germ cell proliferation, thus resulting in $X Y$ sex reversal.

Detailed understanding of the molecular mechanisms underlying the sex reversal in the $X Y$ hybrids requires isolation and molecular characterization of the $\mathrm{Hml}$ locus. Although we found 12 candidate genes including 7 zinc finger proteins within the $\mathrm{Hml}$ region, other genes should be potentially located on the gap regions. To find them, we performed genomic synteny analysis using the Genomicus genome browser (version 60.01; http:// www.dyogen.ens.fr/genomicus/). However, we could not find other candidate genes by this approach, because the genes around the $\mathrm{Hml}$ region were mapped to several chromosomes and/or scaffolds in other fish species including stickleback and Tetraodon. Furthermore, the gene order was not conserved among these fish species. Therefore, we have taken a positional approach to isolate the $\mathrm{Hml}$ gene, and started a chromosome walking using a bacterial artificial chromosome clones. So far, we have isolated two bacterial artificial chromosome clones that fill the gaps, and estimated the $1.2 \mathrm{~cm}$ region of interest to be $670-720 \mathrm{~kb}$ (with the gap region as $200-250 \mathrm{~kb}$ in total) which is similar to that in the genome data. To identify the $\mathrm{Hml}$ gene, we will determine the complete genomic sequence of these bacterial artificial chromosome clones, identify the functional genes in this genomic region and perform functional analyses of the candidate genes. Isolation of the $\mathrm{Hml}$ gene will help to define not only the processes and mechanisms underlying the hybrid $X Y$ sex reversal but also the transcriptional regulatory mechanism of Dmy in normal development of O. latipes and O. curvinotus.

\section{Conflict of interest}

The authors declare no conflict of interest.

\section{Acknowledgements}

This work was supported in part by Grants-in-Aid for Scientific Research on Priority Areas from the Ministry of Education, Culture, Sports, Science and Technology of Japan to S.H. (17052007, 19040009 and 21570221).

\section{References}

Aida T (1921). On the inheritance of color in a freshwater fish, Aplocheilus latipes Temmick and Schlegel, with special reference to sex-linked inheritance. Genetics 6: 554-573.

Capel B, Rasberry C, Dyson J, Bishop CE, Simpson E, Vivian N et al. (1993). Deletion of $Y$ chromosome sequences located outside the testis determining region can cause $\mathrm{XY}$ female sex reversal. Nat Genet 5: 301-307.

Eicher EM, Washburn LL, Schork NJ, Lee BK, Shown EP, Xu X et al. (1996). Sex-determining genes on mouse autosomes identified by linkage analysis of $\mathrm{C} 57 \mathrm{BL} / 6 \mathrm{~J}-\mathrm{Y}^{\mathrm{POS}}$ sex reversal. Nat Genet 14: 206-209.

Eicher EM, Washburn LL, Whitney III JB, Morrow KE (1982). Mus poschiavinus $\mathrm{Y}$ chromosome in the C57BL/6J murine genome causes sex reversal. Science 217: 535-537.

Gubbay J, Collignon J, Koopman P, Capel B, Economou A, Munsterberg A et al. (1990). A gene mapping to the sexdetermining region of the mouse $\mathrm{Y}$ chromosome is a member of a novel family of embryonically expressed genes. Nature 346: 245-250.

Hyodo-Taguchi Y (1996). Inbred strains of the medaka, Oryzias latipes. Fish Biol J Medaka 8: 11-14.

Kato M, Takehana Y, Sakaizumi M, Hamaguchi S (2010). A sexdetermining region on the $\mathrm{Y}$ chromosome controls the sexreversal ratio in interspecific hybrids between Oryzias curvinotus females and Oryzias latipes males. Heredity 104: 191-195.

Kimura T, Jindo T, Narita T, Naruse K, Kobayashi D, Shin-I T et al. (2004). Large-scale isolation of ESTs from medaka embryos and its application to medaka developmental genetics. Mech Dev 121: 915-932.

Kinoshita M, Murata K, Naruse K, Tanaka M (2009). Medaka: Biology, Management and Experimental Protocols. Wiley-Blackwell: Iowa.

Kobayashi T, Matsuda M, Kajiura-Kobayashi H, Suzuki A, Saito $\mathrm{N}$, Nakamoto $\mathrm{M}$ et al. (2004). Two DM domain genes, DMY and DMRT1, involved in testicular differentiation and development in the medaka, Oryzias latipes. Dev Dyn 231: 518-526.

Kondo M, Nagao E, Mitani H, Shima A (2001). Differences in recombination frequencies during female and male meioses of the sex chromosomes of the medaka, Oryzias latipes. Genet Res 78: 23-30.

Koopman P, Gubbay J, Vivian N, Goodfellow P, Lovell-Badge R (1991). Male development of chromosomally female mice transgenic for Sry. Nature 351: 117-121.

Matsuda M, Nagahama Y, Shinomiya A, Sato T, Matsuda C, Kobayashi T et al. (2002). DMY is a Y-specific DM-domain gene required for male development in the medaka fish. Nature 417: 559-563.

Matsuda M, Sato T, Toyazaki Y, Nagahama Y, Hamaguchi S, Sakaizumi M (2003). Oryzias curvinotus has DMY, a gene that is required for male development in the medaka, O. latipes. Zool Sci 20: 159-161.

Matsuda M, Shinomiya A, Kinoshita M, Suzuki A, Kobayashi T, Paul-Prasanth B et al. (2007). The DMY gene induces male development in genetically female (XX) medaka fish. Proc Natl Acad Sci USA 104: 3865-3870.

Munger SC, Aylor DL, Syed HA, Magwene PM, Threadgill DW, Capel B (2009). Elucidation of the transcription network governing mammalian sex determination by exploiting strain-specific susceptibility to sex reversal. Genes Dev 23: 2521-2536. 
Nanda I, Kondo M, Hornung U, Asakawa S, Winkler C, Shimizu A et al. (2002). A duplicated copy of DMRT1 in the sex-determining region of the Y chromosome of the medaka, Oryzias latipes. Proc Natl Acad Sci USA 99: 11778-11783.

Nikolova G, Sinsheimer JS, Eicher EM, Vilain E (2008). The chromosome 11 region from strain 129 provides protection from sex reversal in XYPOS mice. Genetics 179: 419-427.

Otake H, Hayashi Y, Hamaguchi S, Sakaizumi M (2008). The Y chromosome that lost the male-determining function behaves as an X chromosome in the medaka fish, Oryzias latipes. Genetics 179: 2157-2162.

Otake H, Masuyama H, Mashima Y, Shinomiya A, Myosho T, Nagahama Y et al. (2009). Heritable artificial sex chromosomes in the medaka, Oryzias latipes. Heredity 105: 247-256.

Otake H, Shinomiya A, Matsuda M, Hamaguchi S, Sakaizumi $M$ (2006). Wild-derived $X Y$ sex-reversal mutants in the medaka, Oryzias latipes. Genetics 173: 2083-2090.

Sakaizumi M, Shimizu Y, Hamaguchi S (1992). Electrophoretic studies of meiotic segregation in inter- and intraspecific hybrids among east Asian species of the genus Oryzias (Pisces: Oryziatidae). J Exp Zool 264: 85-92.
Sekido R, Lovell-Badge R (2008). Sex determination involves synergistic action of SRY and SF1 on a specific Sox 9 enhancer. Nature 453: 930-934.

Shinomiya A, Kato M, Yaezawa M, Sakaizumi M, Hamaguchi S (2006). Interspecific hybridization between Oryzias latipes and Oryzias curvinotus causes XY sex reversal. J Exp Zool 305: 890-896.

Shinomiya A, Otake H, Togashi K, Hamaguchi S, Sakaizumi M (2004). Field survey of sex-reversals in the medaka, Oryzias latipes: genotypic sexing of wild populations. Zool Sci 21: 613-619.

Sinclair AH, Berta P, Palmer MS, Hawkins JR, Griffiths BL, Smith MJ et al. (1990). A gene from the human sexdetermining region encodes a protein with homology to a conserved DNA-binding motif. Nature 346: 240-244.

Takehana Y, Naruse K, Sakaizumi M (2005). Molecular phylogeny of the medaka fishes genus Oryzias (Beloniformes: Adrianichthyidae) based on nuclear and mitochondrial DNA sequences. Mol Phylogenet Evol 36: 417-428.

Wilhelm D, Palmer S, Koopman P (2007). Sex determination and gonadal development in mammals. Physiol Rev 87: 1-28.

Supplementary Information accompanies the paper on Heredity website (http://www.nature.com/hdy) 